\title{
Quantum-classical transition of the escape rate of uniaxial antiferromagnetic particles in an arbitrarily directed field
}

\author{
Bin Zhou, ${ }^{1-3}$ Ruibao Tao, ${ }^{1}$ and Shun-Qing Shen ${ }^{2}$ \\ ${ }^{1}$ Department of Physics, Fudan University, Shanghai 200433, China \\ ${ }^{2}$ Department of Physics, The University of Hong Kong, Hong Kong, China \\ ${ }^{3}$ Department of Physics, Hubei University, Wuhan 430062, China
}

(Received 12 May 2003; published 18 December 2003)

\begin{abstract}
Quantum-classical escape rate transition has been studied for uniaxial antiferromagnetic particles with an arbitrarily directed magnetic field. In the case that the transverse and longitudinal fields coexist, we calculate the phase boundary line between first- and second-order transitions, from which phase diagrams can be obtained. It is shown that the effects of the applied longitudinal magnetic field on quantum-classical transition vary greatly for different relative magnitudes of the noncompensation.
\end{abstract}

DOI: 10.1103/PhysRevB.68.214423

PACS number(s): 75.50.Xx, 75.45.+j, 03.65.Sq

Escape from a stable or metastable state at high temperatures is governed by a classical thermal activation rate. At low temperatures close to zero, quantum tunneling becomes relevant. When these two escape rates are equal there exists a crossover temperature $T_{0}$ at which a transition between classical and quantum regimes occurs. The study of the quantum-classical transition is an interesting subject with a long history. ${ }^{1}$ One of the main issues in this subject is to determine whether the transition is first or second order. The transition was recognized as a smooth second-order one in the quantum-mechanical models of Affleck ${ }^{1}$ and the cosmological models of Linde. ${ }^{2}$ However, it was shown ${ }^{3}$ that the smooth transition is not generic. Chudnovsky has suggested that the order of transition is determined by the behavior of the Euclidean time oscillation period $\tau(E)$, where $E$ is the energy near the bottom of the Euclidean potential, which corresponds to the top of the potential barrier. ${ }^{3}$ The nonmonotonic behavior of the oscillation period as a function of energy, i.e., the existence of a minimum in the $\tau \sim E$ curve, was proposed as a condition for the first-order transition in quantum-mechanical tunneling. ${ }^{3}$ Later, a sufficient criterion for the first-order phase transition was obtained by carrying out the nonlinear perturbation near the sphaleron solution. ${ }^{4}$

Since the first- and second-order transitions between the quantum and classical behaviors of the escape rates in spin systems were introduced by Chudnovsky and Garanin, 5,6 the topic has attracted considerable attention. ${ }^{7-15}$ Most theoretical studies have been focused on the ferromagnetic particles. However, most ferromagnetic systems are actually ferrimagnetic particles. For instance, both $\mathrm{Mn}_{12} \mathrm{Ac}$ and $\mathrm{Fe}_{8}$ are characterized by a large spin ground state which originates from incomplete compensation of antiferromagnetically coupled spins. ${ }^{16}$ The strong exchange interaction should be taken into consideration. In Ref. 17 Kim treated the phase transition in ferrimagnetic or antiferromagnetic particles for two general forms of the magnetic anisotropy energy. Very recently, the quantum-classical transition in antiferromagnetic particles with biaxial symmetry in the presence of an applied magnetic field along the medium axis or along the easy axis was investigated. ${ }^{18,19}$ Note that recent work of Chudnovsky and Garanin postulates dislocations as the main source of spin tunneling in $\mathrm{Mn}_{12}$ crystals. $^{20}$ Their theory shows that when the external magnetic field is applied along the $c$ axis of the crystal, local rotations of the magnetic anisotropy axis due to dislocations result in the effective local transverse magnetic field. Experimental evidence of the effects of dislocations on tunneling has been also reported..$^{21-24}$ Therefore in the study of the quantum-classical transition of $\mathrm{Mn}_{12}$, the case of coexistence of the transverse and longitudinal magnetic field is worth investigating. Considering the molecular cluster $\mathrm{Mn}_{12}$ actually is ferrimagnetic, the exchange interaction should be also taken into account. In this paper we aim to investigate the quantum-classical transition of the escape rate of uniaxial antiferromagnetic particles in an arbitrarily directed field, i.e., the coexistence of the transverse and longitudinal magnetic field. It is shown that the effects of the applied longitudinal magnetic field on the quantum-classical transition vary greatly for different relative magnitudes of the noncompensation.

We consider a small uniaxial antiferromagnetic particle with two magnetic sublattices whose magnetizations $\mathbf{m}_{1}$ and $\mathbf{m}_{2}$ are coupled by the strong exchange interaction $\mathbf{m}_{1}$ $\cdot \mathbf{m}_{2} / \chi_{\perp}$, where $\chi_{\perp}$ is the perpendicular susceptibility. The system of interest has a noncompensation of sublattice with $m\left(=m_{1}-m_{2}>0\right)$, and easy-axis anisotropy along the $z$ axis. In the presence of an arbitrarily directed magnetic field, i.e., the coexistence of a transverse magnetic field $H_{x}$ along the $x$ axis and a longitudinal one $H_{z}$ along the $z$ axis, the Euclidean action is written as ${ }^{25}$

$$
\begin{aligned}
S_{E}(\theta, \phi)= & V \int d \tau\left(i \frac{m_{1}+m_{2}}{\gamma} \dot{\phi}-i \frac{m}{\gamma} \dot{\phi} \cos \theta+\frac{\tilde{\chi}_{\perp}}{2 \gamma^{2}}[(\dot{\theta}\right. \\
& \left.+i \gamma H_{x} \sin \phi\right)^{2}+\left(\dot{\phi} \sin \theta+i \gamma H_{x} \cos \theta \cos \phi\right. \\
& \left.\left.-i \gamma H_{z} \sin \theta\right)^{2}\right]+K_{\|} \sin ^{2} \theta-m H_{x} \sin \theta \cos \phi \\
& \left.-m H_{z} \cos \theta\right),
\end{aligned}
$$

where $V$ is the volume of the particle, $\gamma$ the gyromagnetic ratio, $\tilde{\chi}_{\perp}=\chi_{\perp}\left(m_{2} / m_{1}\right)$, and $K_{\|}$the longitudinal anisotropy. The polar coordinate $\theta$ and the azimuthal coordinate $\phi$, which are the angular components of $\mathbf{m}_{1}$ in the spherical 


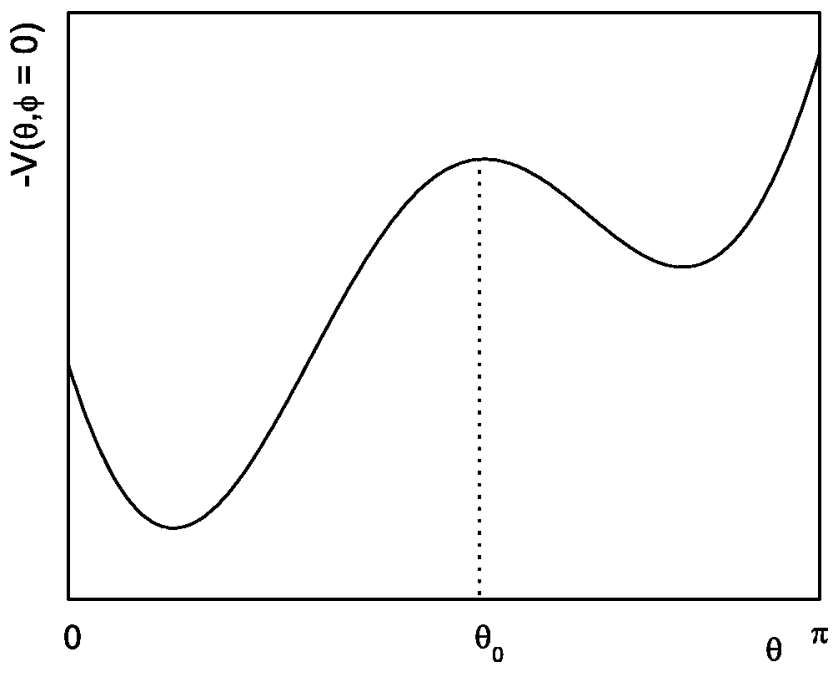

FIG. 1. The effective potential $-V(\theta, \phi=0)$.

coordinate system, can determine the direction of the Néel vector. A dot over a symbol denotes a derivative with respect to the Euclidean time $\tau$.

The classical trajectory corresponding to the Euclidean action (1) is determined by the equations

$$
\begin{aligned}
\text { in } \dot{\phi} \sin \theta+x\left(-i b_{z} \dot{\phi} \sin 2 \theta-2 i b_{x} \dot{\phi} \cos \phi \sin ^{2} \theta-\ddot{\theta}\right. \\
\left.+\frac{1}{2} \dot{\phi}^{2} \sin 2 \theta\right)-\frac{V_{\theta}}{K_{\|}}=0,
\end{aligned}
$$

$-i n \dot{\theta} \sin \theta+x\left(i b_{z} \dot{\theta} \sin 2 \theta+2 i b_{x} \dot{\theta} \cos \phi \sin ^{2} \theta-\ddot{\phi} \sin ^{2} \theta\right.$

$$
-\dot{\phi} \dot{\theta} \sin 2 \theta)-\frac{V_{\phi}}{K_{\|}}=0,
$$

where $n=m /\left(K_{\|} \gamma\right), \quad x=\tilde{\chi}_{\perp} /\left(K_{\|} \gamma^{2}\right), \quad b_{x(z)}=\gamma H_{x(z)}, \quad V_{\theta}$ $=\partial V / \partial \theta$ and $V_{\phi}=\partial V / \partial \phi$. The inverted potential is

$$
\begin{aligned}
V(\theta, \phi)= & K_{\|}\left(-\sin ^{2} \theta+2 h_{x} \sin \theta \cos \phi+2 h_{z} \cos \theta\right. \\
& +\frac{x b_{x}^{2}}{2} \sin ^{2} \phi+\frac{x b_{x}^{2}}{2} \cos ^{2} \theta \cos ^{2} \phi+\frac{x b_{z}^{2}}{2} \sin ^{2} \theta \\
& \left.-x b_{x} b_{z} \sin \theta \cos \theta \cos \phi\right)
\end{aligned}
$$

where $h_{x(z)}=H_{x(z)} / H_{c}$ and $H_{c}=2 K_{\|} / m$. In Fig. 1 the effective potential $-V(\theta, \phi=0)$ is drawn. The minima of the potential correspond to the equilibrium orientation of the Néel vector. The metastability condition that $d V(\theta, 0) / d \theta$ $=0$ and $d^{2} V(\theta, 0) / d \theta^{2}=0$ determines the critical parameters at which the barrier vanishes.

In the high-temperature regime the sphaleron solution of Eqs. (2) and (3) is $\left(\theta_{0}, \phi_{0}=0\right) . \theta_{0}$ is the position of the top of the potential barrier $-V\left(\theta_{0}, 0\right)$, and is determined by $d V(\theta, 0) /\left.d \theta\right|_{\theta_{0}}=0$ and $d^{2} V(\theta, 0) /\left.d \theta^{2}\right|_{\theta_{0}}>0 . \theta_{0}$ has a cumbersome analytical form and its numerical result will be applied below to determine phase boundary lines. Furthermore, its behavior is illustrated in Fig. 2 for given $h_{x}$ and $y$. Above,

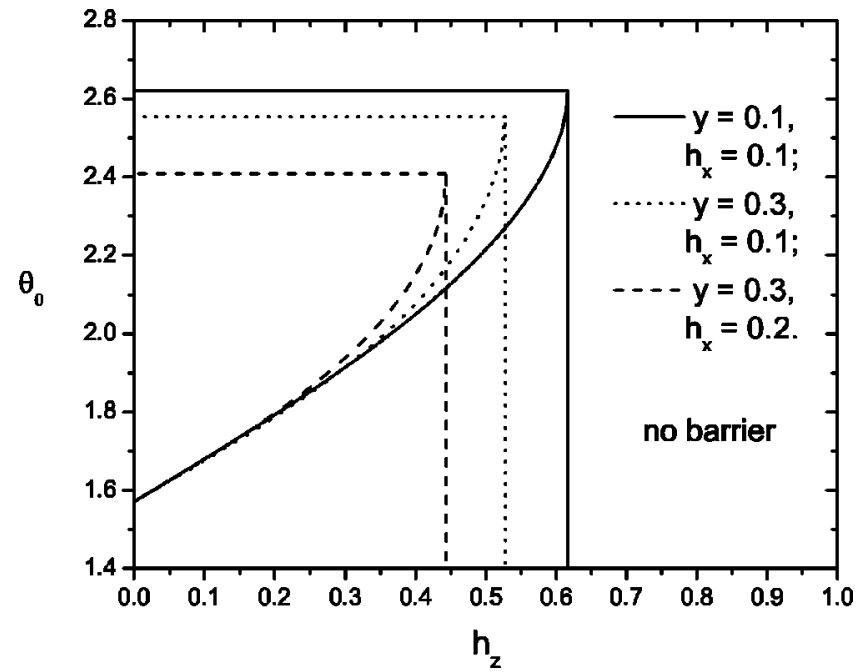

FIG. 2. $\theta_{0}$ versus $h_{z}$ for some given $h_{x}$ and $y$.

$y=x / n^{2}\left(=\tilde{\chi}_{\perp} K_{\|} / m^{2}\right)$ and the parameter $y$ indicates the relative magnitude of the noncompensation. For large noncompensation $\left(y \ll 1\right.$, i.e., $\left.m \gg \sqrt{\tilde{\chi}_{\perp} K_{\|}}\right)$and for small noncompensation $\left(y \gg 1\right.$, i.e., $m \ll \sqrt{\tilde{\chi}_{\perp} K_{\|}}$), the system becomes ferromagnetic and nearly compensated antiferromagnetic, respectively. ${ }^{17}$ Note that $\theta_{0}=\pi / 2$ for $h_{z}=0$. The crossover behavior of the escape rate of this model from quantum tunneling to thermal activation can be obtained from the deviation of the period of the periodic instanton from that of the sphaleron. To this end we expand $(\theta, \phi)$ about the sphaleron configurations $\theta_{0}$ and $\phi_{0}$, i.e., $\theta=\theta_{0}+\eta(\tau)$ and $\phi=\phi_{0}$ $+\xi(\tau)$, where $\phi_{0}=0$. Substituting them into Eqs. (2) and (3) one yields the following power series equations of the fluctuation fields $\eta$ and $\xi$ :

$$
\left(\begin{array}{l}
G_{1}^{\xi}(\eta, \xi) \\
G_{1}^{\eta}(\eta, \xi)
\end{array}\right)+\left(\begin{array}{c}
G_{2}^{\xi}(\eta, \xi) \\
G_{2}^{\eta}(\eta, \xi)
\end{array}\right)+\left(\begin{array}{l}
G_{3}^{\xi}(\eta, \xi) \\
G_{3}^{\eta}(\eta, \xi)
\end{array}\right)+\cdots=0,
$$

where $G_{1}, G_{2}, G_{3}, \ldots$ denote terms which contain linear, quadratic, cubic, and higher powers of the small fluctuations, respectively:

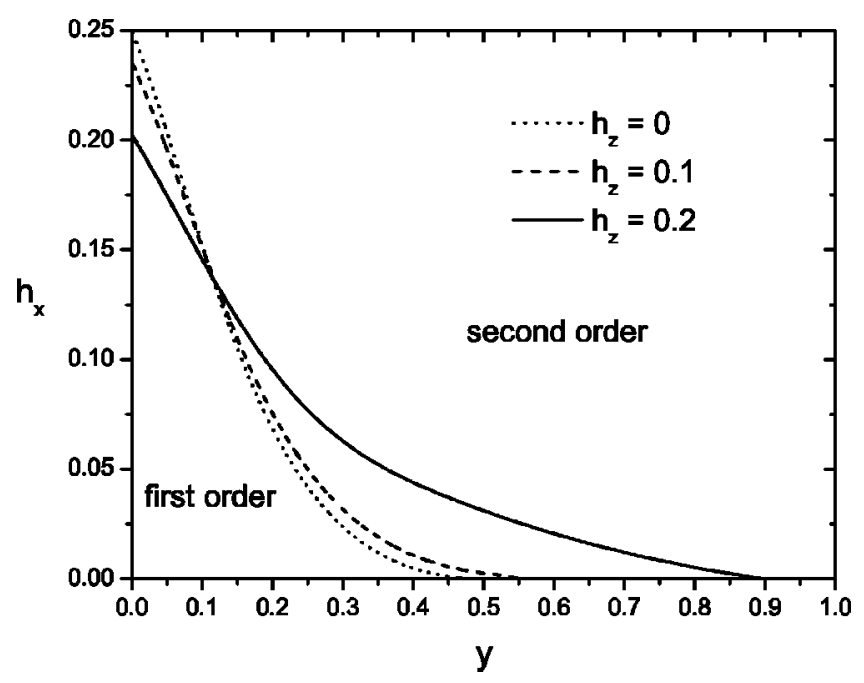

FIG. 3. Phase diagram $h_{x}(y)$ for $h_{z}=0,0.1$, and 0.2. 


$$
\begin{aligned}
& G_{1}^{\xi}(\eta, \xi)=i n \sin \theta_{0} \dot{\xi}-i x\left(b_{z} \sin 2 \theta_{0}+2 b_{x} \sin ^{2} \theta_{0}\right) \dot{\xi}-x \ddot{\eta} \\
& +A_{1} \eta \\
& G_{2}^{\xi}(\eta, \xi)=i n \cos \theta_{0} \eta \dot{\xi}-2 i x\left(b_{x} \sin 2 \theta_{0}+b_{z} \cos 2 \theta_{0}\right) \eta \dot{\xi} \\
& +\frac{1}{2} x \sin 2 \theta_{0} \xi^{2}+A_{2} \xi^{2}+A_{4} \eta^{2} \\
& G_{3}^{\xi}(\eta, \xi)=-\frac{i}{2} n \sin \theta_{0} \eta^{2} \dot{\xi}+2 i x\left(b_{z} \sin 2 \theta_{0}-b_{x} \cos 2 \theta_{0}\right) \eta^{2} \dot{\xi} \\
& +i x b_{x} \sin ^{2} \theta_{0} \xi^{2} \dot{\xi}+x \cos 2 \theta_{0} \eta \dot{\xi}^{2}+A_{3} \eta \xi^{2} \\
& +A_{5} \eta^{3} \\
& G_{1}^{\eta}(\eta, \xi)=i n \sin \theta_{0} \dot{\eta}-i x\left(b_{z} \sin 2 \theta_{0}+2 b_{x} \sin ^{2} \theta_{0}\right) \dot{\eta} \\
& +x \sin ^{2} \theta_{0} \ddot{\xi}+B_{1} \xi \\
& G_{2}^{\eta}(\eta, \xi)=i n \cos \theta_{0} \eta \dot{\eta}-2 i x\left(b_{x} \sin 2 \theta_{0}+b_{z} \cos 2 \theta_{0}\right) \eta \dot{\eta} \\
& +x \sin 2 \theta_{0}(\dot{\eta} \dot{\xi}+\eta \ddot{\xi})+B_{2} \eta \xi \\
& G_{3}^{\eta}(\eta, \xi)=-\frac{1}{2} i n \sin \theta_{0} \eta^{2} \dot{\eta}+2 i x\left(b_{z} \sin 2 \theta_{0}\right. \\
& \left.-b_{x} \cos 2 \theta_{0}\right) \eta^{2} \dot{\eta}+i x b_{x} \sin ^{2} \theta_{0} \xi^{2} \dot{\eta} \\
& +x \cos 2 \theta_{0}\left(2 \eta \dot{\eta} \dot{\xi}+\eta^{2} \ddot{\xi}\right)+B_{3} \eta^{2} \xi+B_{4} \xi^{3}
\end{aligned}
$$

where

$$
\begin{gathered}
A_{1}=-\frac{V_{\theta \theta}}{K_{\|}}, \quad A_{2}=-\frac{V_{\theta \phi \phi}}{2 K_{\|}}, \quad A_{3}=-\frac{V_{\theta \theta \phi \phi}}{2 K_{\|}}, \\
A_{4}=-\frac{V_{\theta \theta \theta}}{2 K_{\|}}, \quad A_{5}=-\frac{V_{\theta \theta \theta \theta}}{6 K_{\|}}, \\
B_{1}=\frac{V_{\phi \phi}}{K_{\|}}, B_{2}=\frac{V_{\theta \phi \phi}}{K_{\|}}, B_{3}=\frac{V_{\theta \theta \phi \phi}}{2 K_{\|}}, B_{4}=\frac{V_{\phi \phi \phi \phi}}{6 K_{\|}} .
\end{gathered}
$$

It is introduced that $V_{\theta \theta}=\left[\partial^{2} V / \partial \theta^{2}\right]_{\theta=\theta_{0}, \phi=\phi_{0}}, V_{\theta \phi \phi}$ $=\left[\partial^{2} V / \partial \theta \partial \phi^{2}\right]_{\theta=\theta_{0}, \phi=\phi_{0}}$, and so on.

Denoting $\delta \Omega(\tau) \equiv(\eta(\tau), \xi(\tau))$, we have $\delta \Omega(\tau+\beta \hbar)$ $=\delta \Omega(\tau)$ at finite temperature and write it as the Fourier series $\delta \Omega(\tau)=\sum_{n=-\infty}^{\infty} \delta \Omega_{n} \exp \left[i \omega_{n} \tau\right]$, where $\omega_{n}=2 \pi n / \beta \hbar$. Since simple analysis shows that $\eta$ is real and $\xi$ imaginary, to the lowest order we write them in the form $\eta$ $\simeq a \theta_{1} \cos (\omega \tau)$ and $\xi \simeq i a \phi_{1} \sin (\omega \tau)$. Here $a$ serves as a perturbation parameter. Substituting them into Eq. (5) and neglecting terms of order higher than $a$, we obtain the relation

$$
\begin{aligned}
\frac{\phi_{1}}{\theta_{1}} & =\frac{x \omega_{ \pm}^{2}+A_{1}}{\omega_{ \pm}\left[n-2 x\left(b_{z} \cos \theta_{0}+b_{x} \sin \theta_{0}\right)\right] \sin \theta_{0}} \\
& =-\frac{\omega_{ \pm}\left[n-2 x\left(b_{z} \cos \theta_{0}+b_{x} \sin \theta_{0}\right)\right] \sin \theta_{0}}{x \omega_{ \pm}^{2} \sin ^{2} \theta_{0}-B_{1}}
\end{aligned}
$$

and the oscillation frequency

$$
\begin{aligned}
\omega_{ \pm}^{2}= & -\frac{1}{2 x^{2}}\left\{\left(A_{1}-B_{1} \csc ^{2} \theta_{0}\right) x+\left[n-2 x\left(b_{z} \cos \theta_{0}+b_{x} \sin \theta_{0}\right)\right]^{2}\right\} \\
& \pm \frac{1}{2 x^{2}} \sqrt{4 A_{1} B_{1} x^{2} \csc ^{2} \theta_{0}+\left\{\left(A_{1}-B_{1} \csc ^{2} \theta_{0}\right) x+\left[n-2 x\left(b_{z} \cos \theta_{0}+b_{x} \sin \theta_{0}\right)\right]^{2}\right\}^{2}} .
\end{aligned}
$$

Next, let us write $\eta \simeq a \theta_{1} \cos (\omega \tau)+\eta_{2}$, and $\xi$ $\simeq i a \phi_{1} \sin (\omega \tau)+i \xi_{2}$, where $\eta_{2}$ and $\xi_{2}$ are of the order of $a^{2}$. Inserting them into Eq. (5), we arrive at $\omega=\omega_{+}$and

$$
\eta_{2}=a^{2} p_{0}+a^{2} p_{2} \cos (2 \omega \tau), \quad \xi_{2}=a^{2} q_{2} \sin (2 \omega \tau)
$$

where the analytic forms of coefficients $p_{0}, p_{2}$, and $q_{2}$ are cumbersome, which are listed in the Appendix.

This implies that there is no shift in the oscillation frequency. In order to find the change of the oscillation period, we proceed to the third order of perturbation theory by writing $\eta \simeq a \theta_{1} \cos (\omega \tau)+\eta_{2}+\eta_{3}$, and $\xi \simeq i a \phi_{1} \sin (\omega \tau)+i \xi_{2}+i \xi_{3}$, where $\eta_{3}$ and $\xi_{3}$ are of the order of $a^{3}$. Substituting them again into Eq. (5), and retaining only the terms up to $O\left(a^{3}\right)$, we have

$$
n^{4} y^{2}\left(\omega^{2}-\omega_{+}^{2}\right)\left(\omega^{2}-\omega_{-}^{2}\right)=a^{2} \frac{\theta_{1}^{2}}{4 \sin ^{2} \theta_{0}} g\left(h_{x}, h_{z}, y\right),
$$

where

$$
g\left(h_{x}, h_{z}, y\right)=g_{1}\left(h_{x}, h_{z}, y\right)+g_{2}\left(h_{x}, h_{z}, y\right) .
$$

The forms of $g_{1}\left(h_{x}, h_{z}, y\right)$ and $g_{2}\left(h_{x}, h_{z}, y\right)$ are 


$$
\begin{aligned}
g_{1}\left(h_{x}, h_{z}, y\right)= & -\left(A_{1}+y w^{2}\right)\left[3 B_{4} \lambda^{2}-6 \lambda h_{x} w \sin ^{2} \theta_{0}-B_{3}-2 B_{2}\left(2 \tilde{p}_{0}-\tilde{p}_{2}\right)+3 y w^{2} \cos 2 \theta_{0}+2 y w^{2} \sin 2 \theta_{0}\left(2 \tilde{p}_{0}+\tilde{p}_{2}\right)\right. \\
& +\frac{1}{\lambda}\left(-2 B_{2} \tilde{q}_{2}+2 w \cos \theta_{0}\left(2 \tilde{p}_{0}+\tilde{p}_{2}\right)-4 h_{x} y w \cos 2 \theta_{0}-\frac{1}{2} w \sin \theta_{0}-8 h_{z} y w\left(2 \tilde{p}_{0}+\tilde{p}_{2}\right) \cos 2 \theta_{0}\right. \\
& \left.\left.+4 y w \sin 2 \theta_{0}\left(h_{z}-4 h_{x} \tilde{p}_{0}-2 h_{x} \tilde{p}_{2}\right)+4 \tilde{q}_{2} y w^{2} \sin 2 \theta_{0}\right)\right], \\
g_{2}\left(h_{x}, h_{z}, y\right)= & \left(B_{1}-y w^{2} \sin ^{2} \theta_{0}\right)\left[2 \lambda^{3} h_{x} y w \sin ^{2} \theta_{0}-\lambda^{2}\left(A_{3}+3 y w^{2} \cos 2 \theta_{0}\right)+\lambda\left(-4 A_{2} \tilde{q}_{2}-2\left(2 \tilde{p}_{0}+\tilde{p}_{2}\right) w \cos \theta_{0}\right.\right. \\
+ & \left.\frac{3}{2} w \sin \theta_{0}-4 \tilde{q}_{2} y w^{2} \sin 2 \theta_{0}+4 y w \cos 2 \theta_{0}\left(3 h_{x}+4 h_{z} \tilde{p}_{0}+2 h_{z} \tilde{p}_{2}\right)-4 y w \sin 2 \theta_{0}\left(3 h_{z}-4 h_{x} \tilde{p}_{0}-2 h_{x} \tilde{p}_{2}\right)\right) \\
+ & \left.4 A_{4}\left(2 \tilde{p}_{0}+\tilde{p}_{2}\right)+4 \tilde{q}_{2} w\left(4 h_{z} y \cos 2 \theta_{0}+4 h_{x} y \sin 2 \theta_{0}-\cos \theta_{0}\right)+3 A_{5}\right]
\end{aligned}
$$

where $\quad w=n \omega_{+}$and $\lambda=\phi_{1} / \theta_{1}$. Again, $y=x / n^{2}$ $\left(=\tilde{\chi}_{\perp} K_{\|} / m^{2}\right)$ and the parameter $y$ indicates the relative magnitude of the noncompensation. Also, $\tilde{p}_{0}, \tilde{p}_{2}$, and $\tilde{q}_{2}$ are obtained by replacing $\phi_{1}$ by $\lambda \theta_{1}$ and dropping $\theta_{1}^{2}$ in $p_{0}, p_{2}$, and $q_{2}$, respectively. It can be shown that for $h_{z}=0$ Eq. (12) is reduced to the case corresponding to uniaxial antiferromagnetic particle with a transverse magnetic field only has been investigated in Ref. 17.

As shown by Chudnovsky, ${ }^{3}$ if the oscillation period $\tau$ is not a monotonic function of $a$, where $a$ is a function of $E$ in the absence of dissipation, the system exhibits a first-order transition. Thus the period $\tau(=2 \pi / \omega)$ in Eq. (11) should be less than $\tau_{+}\left(=2 \pi / \omega_{+}\right)$, i.e., $\omega>\omega_{+}$for the first-order transition. It implies that $g\left(h_{x}, h_{z}, y\right)>0$ in Eq. (11) for the firstorder transition, and $g\left(h_{x}, h_{z}, y\right)=0$ determines the phase boundary between the first- and the second-order transition. In this case the three parameters $h_{x}, h_{z}, y$ should be treated simultaneously, which is not a simple problem. In the present work we will fix one parameter and then compute the boundary curve with the other two parameters. We first solve Eq. (11) numerically to obtain the phase boundary lines $h_{x}(y)$ 's for several values of $h_{z}$, which are plotted in Fig. 3. From Fig. 3, an immediate observation is that the first-order region for a given $h_{z}$ diminishes as $y$ increases, which shows the same trend as the $h_{z}=0$ case. Thus it is evident that the region for the first-order transition is greatly reduced as the system becomes ferrimagnetic and there is no first-order transition in almost compensated antiferromagnetic particles. The result coincides with Ref. 17. Figure 3 also shows that with increasing $h_{z}$ the variety of first-order region is not a simple case. For the small $y$ case with increasing $h_{z}$ the firstorder region is shrunk, while for the larger $y$ case the longitudinal field $h_{z}$ favors occurrence of the first-order transition. For instance, for the case of $y=0.05$, the maximum values of the transverse field $h_{x}$ for occurrence of the first-order transition are $h_{x}=0.203,0.195$, and 0.175 for $h_{z}=0,0.1$, and 0.2 , respectively. On the other hand, the first-order region vanishes beyond $y \simeq 0.46,0.55$, and 0.89 for $h_{z}=0,0.1$, and 0.2 , respectively. This can be qualitatively understood from the consideration that the height of the effective potential barrier decreases as $h_{z}$ increases, whereas the height increases as $y$ increases, therefore there is a competition between the longitudinal field and the relative magnitude of the noncompensation. When $y=0$, the fact that the region for the first-order transition decreases as the longitudinal field increases results from a flattening of the peak of the barrier. ${ }^{11}$ For the small $y$ case (i.e., the large noncompensation), the crossover behavior of the ferrimagnetic system still keeps qualitatively that of the ferromagnetic one. However, for the larger $y$ case the exchange interaction plays the role of effective magnetic field and so, for a given small transverse field the region for the first-order transition increases as the longitudinal field increases. To illustrate further the effect of the longitudinal field $h_{z}$ on quantum-classical transition, we next calculate the phase boundary lines $h_{x}\left(h_{z}\right)$ 's for several values of $y$, which are shown in Figs. 4 and 5. In Fig. 4, for the case of $y=0$ corresponding to uniaxial ferromagnetic system, the phase boundary line is plotted by a dotted line. Obviously the line coincides with Fig. 13 in Ref. 11, in which quantum-classical transition in a uniaxial ferromagnetic system with a transverse magnetic field and a longitudinal one was investigated. For the case of $y=0.1$, the phase boundary lines $h_{x}\left(h_{z}\right)$ 's shift downwards and with $h_{z}$ increasing the critical value of $h_{x}$ decrease monotonically. Figure 5 gives another case, in which the phase boundary lines $h_{x}\left(h_{z}\right)$ 's show a kind of nonmonotonic behavior. For instance, for the case of $y=0.3$, the first-order region vanishes beyond $h_{x} \simeq 0.024$ for $h_{z}=0$, while the maximum is $h_{x}$ $\simeq 0.112$ for $h_{z}=0.332$.

It was shown that quantum tunneling will show up at higher temperatures and higher frequencies in antiferromagnetic particles than in ferromagnetic particles of similar size. ${ }^{25}$ Moreover, most ferromagnetic systems are ferrimagnetic, so nanometer-scale antiferromagnets are more interesting from experimental and theoretical aspects. But a detailed comparison between the theory and experiment on quantumclassical transition remains a challenging task. It is very important to obtain the information on the magnitude of the quantity $y$ for observing the first-order transition in real ex- 


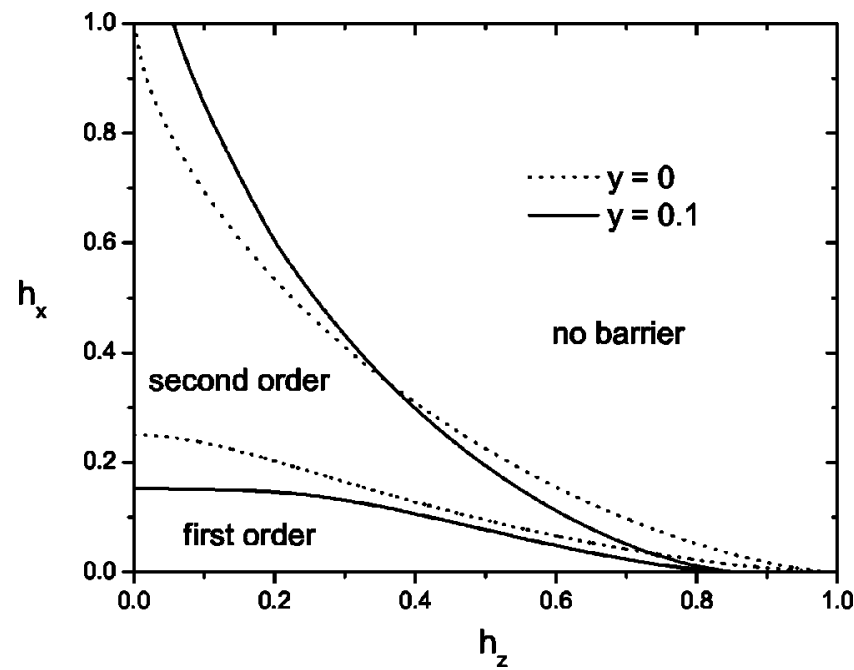

FIG. 4. Phase diagram $h_{x}\left(h_{z}\right)$ for $y=0$ and 0.1 .

periments. For the typical antiferromagnetic particle with $\chi$ $\sim 10^{-4}, K_{\|} \sim 10^{6} \mathrm{erg} / \mathrm{cm}^{3}$, and $m \sim 500 \mathrm{emu} / \mathrm{cm}^{3}$, one can get the quantity $y \simeq 10^{-4}$. ${ }^{19}$ In this case, for the longitudinal field parameter $h_{z}=0.4$, the range of the transverse field parameter for observing the first-order transition is $0<h_{x}$ $\$ 0.127$. It is noted that Wensdorfer et al. have performed the switching field measurements on individual ferrimagnetic and insulating $\mathrm{BaFeCoTiO}$ nanoparticle containing about $10^{5}-10^{6}$ spins at very low temperature $(0.1-6 \mathrm{~K}){ }^{26}$ Below $0.4 \mathrm{~K}$, experimental results are quantitatively in agreement with the predictions of the macroscopic quantum tunneling theory without dissipation. The BaFeCoTiO nanoparticles have a strong uniaxial magnetocrystalline anisotropy. ${ }^{26}$ Therefore the material is expected as a candidate to investigate quantum-classical transition of the escape rate of uniaxial ferrimagnetic or antiferromagnetic particles in an arbitrarily directed field.

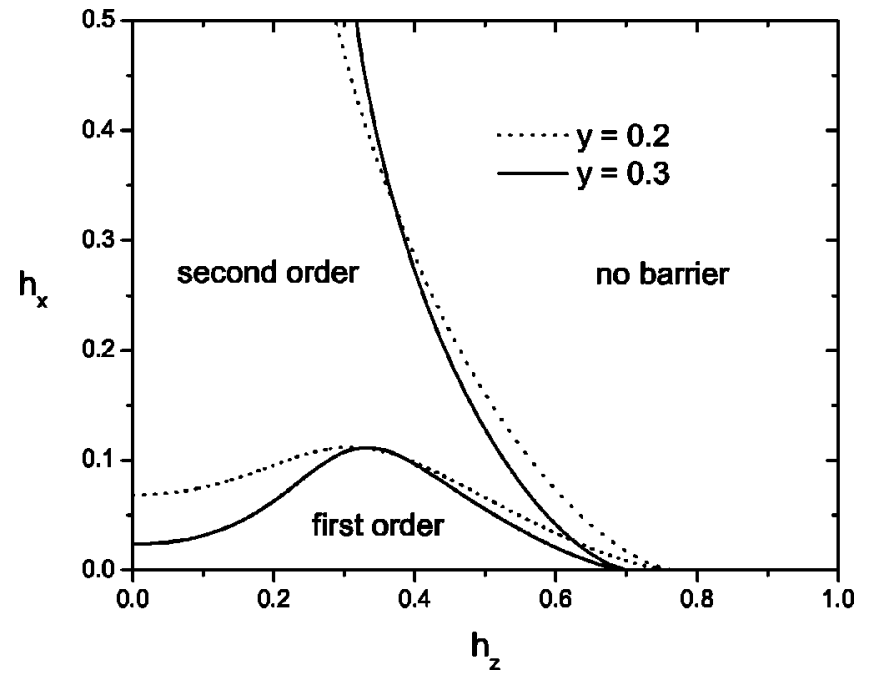

FIG. 5. Phase diagram $h_{x}\left(h_{z}\right)$ for $y=0.2$ and 0.3 .

In conclusion, we have investigated quantum-classical escape rate transition for uniaxial antiferromagnetic particle with an arbitrarily directed magnetic field, i.e., the coexistence of the transverse and longitudinal magnetic fields. There are three parameters which can be controlled by experiment: relative magnitude of the noncompensation and two field parameters. The nonlinear perturbation method is used to obtain various phase diagrams for first- and secondorder transition depending on the three parameters. It is shown that the effects of the applied longitudinal magnetic field on quantum-classical transition vary greatly for different relative magnitudes of the noncompensation.

The work was supported by the China Postdoctoral Science Foundation under Grant No. 2002032138, the National Natural Science Foundation of China under Grant Nos. 10174015 and 10234010, and the Research Grants Council of Hong Kong under Grant No. HKU7023/03P.

\section{APPENDIX}

The coefficients in Eq. (10) are deduced by using the software MATHEMATICA3.0:

$$
\begin{aligned}
& p_{0}=\frac{1}{2 A_{1}}\left[\phi_{1} \cos \theta_{0}\left(n \theta_{1} \omega+x \phi_{1} \omega_{+}^{2} \sin \theta_{0}\right)+A_{2} \phi_{1}^{2}-A_{4} \theta_{1}^{2}-2 x \omega \theta_{1} \phi_{1}\left(b_{z} \cos 2 \theta_{0}+b_{x} \sin 2 \theta_{0}\right)\right], \\
& p_{2}=\left(B_{1}\left(A_{4} \theta_{1}^{2}+A_{2} \phi_{1}^{2}\right)+2 B_{2} n \omega \theta_{1} \phi_{1} \sin \theta_{0}-8 b_{z}^{2} x^{2} \theta_{1}^{2} \omega^{2} \cos ^{3} \theta_{0} \sin \theta_{0}-2 x \omega\left(2 B_{2} b_{x} \theta_{1} \phi_{1}+B_{1} b_{z} \theta_{1} \phi_{1}+2 A_{4} \theta_{1}^{2} \omega+2 A_{2} \phi_{1}^{2} \omega\right)\right. \\
& \times \sin ^{2} \theta_{0}+8 b_{z} x^{2} \theta_{1} \omega^{2}\left(b_{x} \theta_{1}+\omega \phi_{1}\right) \sin ^{4} \theta_{0}-4 b_{z} n x \theta_{1}^{2} \omega^{2} \sin ^{3} \theta_{0}+2 b_{z} x \theta_{1} \omega \cos ^{2} \theta_{0}\left[B_{1} \phi_{1}-6 b_{x} x \theta_{1} \omega-2 x \phi_{1} \omega^{2}+4 x \phi_{1} \omega_{+}^{2}\right. \\
& \left.+2 x\left(3 b_{x} \theta_{1} \omega+\phi_{1} \omega^{2}-2 \phi_{1} \omega_{+}^{2}\right) \cos 2 \theta_{0}+4 n \theta_{1} \omega \sin \theta_{0}\right]-\cos \theta_{0}\left\{B_{1} n \theta_{1} \phi_{1} \omega+\left[2 \theta_{1} \omega\left(n^{2} \theta_{1} \omega+2 B_{2} b_{z} x \phi_{1}\right)\right.\right. \\
& \left.-B_{1} x \phi_{1}\left(4 b_{x} \theta_{1} \omega-\phi_{1} \omega_{+}^{2}\right)\right] \sin \theta_{0}-4 n x \theta_{1} \omega\left[3 b_{x} \theta_{1} \omega+\phi_{1}\left(\omega^{2}-2 \omega_{+}^{2}\right)\right] \sin ^{2} \theta_{0}+4 x^{2} \omega\left[4 b_{x}^{2} \theta_{1}^{2} \omega+4 b_{x} \theta_{1} \phi_{1}\left(\omega^{2}-\omega_{+}^{2}\right)\right. \\
& \left.\left.\left.-\omega\left(2 b_{z}^{2} \theta_{1}^{2}+\phi_{1}^{2} \omega_{+}^{2}\right)\right] \sin ^{3} \theta_{0}\right\}\right) /\left\{-2 B_{1}\left(A_{1}+4 x \omega^{2}\right)+8 \omega^{2}\left[A_{1} x+\left(n-2 b_{z} x \cos \theta_{0}\right)^{2}+4 x^{2} \omega^{2}\right] \sin ^{2} \theta_{0}\right. \\
& \left.+32 b_{x} x \omega^{2}\left(2 b_{z} x \cos \theta_{0}-n\right) \sin ^{3} \theta_{0}+32 b_{x}^{2} x^{2} \omega^{2} \sin ^{4} \theta_{0}\right\} \text {, } \\
& q_{2}=\left\{\theta_{1}\left(A_{1}+4 x \omega^{2}\right)\left[B_{2} \phi_{1}-n \theta_{1} \omega \cos \theta_{0}+2 x \theta_{1} \omega\left(b_{x} \sin 2 \theta_{0}+b_{z} \cos 2 \theta_{0}\right)-2 x \phi_{1} \omega_{+}^{2} \sin 2 \theta_{0}\right]\right. \\
& -\omega \sin \theta_{0}\left[n-2 x\left(b_{z} \cos \theta_{0}+b_{x} \sin \theta_{0}\right)\right]\left[2 n \theta_{1} \phi_{1} \omega \cos \theta_{0}-4 x \theta_{1} \phi_{1} \omega\left(b_{x} \sin 2 \theta_{0}+b_{z} \cos 2 \theta_{0}\right)+x \phi_{1}^{2} \omega_{+}^{2} \sin 2 \theta_{0}-2 A_{4} \theta_{1}^{2}\right. \\
& \left.\left.-2 A_{2} \phi_{1}^{2}\right]\right\} /\left\{8 \omega^{2} \sin ^{2} \theta_{0}\left[n-2 x\left(b_{z} \cos \theta_{0}+b_{x} \sin \theta_{0}\right)\right]^{2}+2\left(A_{1}+4 x \omega^{2}\right)\left(4 x \omega^{2} \sin ^{2} \theta_{0}-B_{1}\right)\right\} \text {. }
\end{aligned}
$$


${ }^{1}$ I. Affleck, Phys. Rev. Lett. 46, 388 (1981).

${ }^{2}$ A.D. Linde, Phys. Lett. 100B, 37 (1981).

${ }^{3}$ E.M. Chudnovsky, Phys. Rev. A 46, 8011 (1992).

${ }^{4}$ D.A. Gorokhov and G. Blatter, Phys. Rev. B 56, 3130 (1997).

${ }^{5}$ E.M. Chudnovsky and D.A. Garanin, Phys. Rev. Lett. 79, 4469 (1997).

${ }^{6}$ D.A. Garanin and E.M. Chudnovsky, Phys. Rev. B 56, 11102 (1997)

${ }^{7}$ H.J.W. Müller-Kirsten, D.K. Park, and J.M.S. Rana, Phys. Rev. B 60, 6662 (1999).

${ }^{8}$ J.-Q. Liang, H.J.W. Müller-Kirsten, D.K. Park, and F. Zimmerschied, Phys. Rev. Lett. 81, 216 (1998).

${ }^{9}$ G.-H. Kim, Phys. Rev. B 59, 11847 (1999).

${ }^{10}$ G.-H. Kim and E.M. Chudnovsky, Europhys. Lett. 52, 681 (2000).

${ }^{11}$ D.A. Garanin, X. Martínez Hidalgo, and E.M. Chudnovsky, Phys. Rev. B 57, 13639 (1998).

${ }^{12}$ D.A. Garanin and E.M. Chudnovsky, Phys. Rev. B 59, 3671 (1999).

${ }^{13}$ X. Martíenz Hidalgo and E.M. Chudnovsky, J. Phys.: Condens. Matter 12, 4243 (2000).

${ }^{14}$ B. Zhou, J.-Q. Liang, and F.-C. Pu, Physica B 301, 180 (2001).

${ }^{15}$ S.Y. Lee, H.J.W. Müller-Kirsten, D.K. Park, and F. Zimmerschied, Phys. Rev. B 58, 5554 (1998).
${ }^{16}$ A. Caneschi, D. Gatteschi, C. Sangregorio, R. Sessoli, L. Sorace, A. Cornia, M.A. Novak, C. Paulsen, and W. Wernsdorfer, J. Magn. Magn. Mater. 200, 182 (1999).

${ }^{17}$ G.-H. Kim, Europhys. Lett. 51, 216 (2000).

${ }^{18}$ B. Zhou, J.-Q. Liang, and F.-C. Pu, Phys. Rev. B 64, 132407 (2001).

${ }^{19}$ G.-H. Kim, Phys. Rev. B 67, 144413 (2003).

${ }^{20}$ E.M. Chudnovsky and D.A. Garanin, Phys. Rev. Lett. 87, 187203 (2001).

${ }^{21}$ K. Park, M.A. Novotny, N.S. Dalal, S. Hill, and P.A. Rikvold, Phys. Rev. B 65, 014426 (2002).

${ }^{22}$ B. Parks, J. Loomis, E. Rumberger, D.N. Hendrickson, and G. Christou, J. Appl. Phys. 91, 7170 (2002).

${ }^{23}$ K.M. Mertes, Y. Suzuki, M.P. Sarachik, Y. Paltiel, H. Shtrikman, E. Zeldov, E. Rumberger, D.N. Hendrickson, and G. Christou, Phys. Rev. Lett. 87, 227205 (2001).

${ }^{24}$ J.M. Hernandez, F. Torres, J. Tejada, and E. Molins, Phys. Rev. B 66, 161407 (2002).

${ }^{25}$ E.M. Chudnovsky, J. Magn. Magn. Mater. 140-144, 1821 (1995); B. Barbara and E.M. Chudnovsky, Phys. Lett. A 145, 205 (1990).

${ }^{26}$ W. Wernsdorfer, E. Bonet Orozco, K. Hasselbach, A. Benoit, D. Mailly, O. Kubo, H. Nakano, and B. Barbara, Phys. Rev. Lett. 79, 4014 (1997). 